\title{
Adult Onset Still's Disease in Tropical Area: Illustration of Diagnostic and Therapeutic Difficulties from 3 Senegaleses Observations
}

\author{
Nafy Ndiaye, Ngoné Diaba Diack, Michel Assane Ndour, Biram Codou Fall, Ghislain De Chacus, \\ Daouda Thioub, Ameth Dieng, Yakham Mohamed Leye, Abdoulaye Leye*
}

Internal Medicine Department, Teaching Hospital of Pikine, Dakar, Senegal

Email: *ablayleye@hotmail.com

How to cite this paper: Ndiaye, N., Diack, N.D., Ndour, M.A., Fall, B.C., De Chacus, G., Thioub, D., Dieng, A., Leye, Y.M. and Leye, A. (2017) Adult Onset Still's Disease in Tropical Area: Illustration of Diagnostic and Therapeutic Difficulties from 3 Senegaleses Observations. Open Journal of Internal Medicine, 7, 135-143.

https://doi.org/10.4236/ojim.2017.74014

Received: October 2, 2017

Accepted: November 13, 2017

Published: November 16, 2017

Copyright (๑) 2017 by authors and Scientific Research Publishing Inc. This work is licensed under the Creative Commons Attribution International License (CC BY 4.0).

http://creativecommons.org/licenses/by/4.0/

C) (i) Open Access

\section{Abstract}

Introduction: The Adult Onset Still's Disease (ASD) is a systemic auto-inflammatory affection of unknown cause seldom described in sub-Saharan Africa. We report 3 observations of ASD illustrating the diagnostic and therapeutic difficulties of this affection in our areas. Observation 1: Our first patient is a 56 years old schoolteacher presenting an ASD in its chronic articular form. She had been followed for an inflammatory arthralgia for 10 years and of the pharyngal pains without exact diagnosis. She presented ASD's criteria of Yamaguchi and of Fautrel. The prednisone was begun at the dose of $0.8 \mathrm{mg} / \mathrm{kg} / \mathrm{day}$ with fast appearance of a progressive muscular weakness. Use of methotrexate at a rate of $15 \mathrm{mg}$ per week, associated with low dose of prednisone was effective in long-term without any flare of the disease so far. Observation 2: Our second patient is a 30 years old dressmaker presenting an ASD in its complicated systemic form of lymphohistiocytic activation syndrome. She validated the criteria of Yamaguchi and Fautrel for ASD. She also presented resistance to corticosteroid therapy. The evolution was marked by a hospital-acquired septicemia and a multi-organ failure leading to death. The diagnosis was retrospectively confirmed after that, with the low level of the glycosylated ferritin serum value. Observation 3: The third patient is a 22 years old Guinean student who presented prolonged fever and inflammatory polyarthralgia without articular deformation. He had been misdiagnosed for ASD with diagnostic wandering of several months. He was treated successfully with prednisone after set up of ASD diagnosis according common criteria. Corticosteroid therapy was stopped after 8 months without any relapse noted so far. Conclusion: Caring for ASD is difficult in our context mainly because of high cost of several explorations needed to set up its exact diagnosis while making differential one. Evolution under corticosteroid therapy is usually favorable but diagnostic delay may lead 
to severe complications and death.

\section{Keywords}

Still's Disease, Diagnosis, Treatment, Tropical Area

\section{Introduction}

The Adult Onset Still's Disease (ASD) is an auto-inflammatory systemic affection of unknown cause [1]. Its diagnosis is often tiresome because no sign is pathognomonic. Also it constitutes a diagnosis of exclusion. This diagnosis is made more difficult in tropical context where the presence of fever makes confusion with the endemic infectious causes. Also, the access to the key complementary examinations allowing the diagnosis of ASD is not always easy. It is what explains certainly the low number of cases of ASD brought back in sub-Saharan Africa [2] [3].

Caring for this affection is not facilitated because of lake of codified treatment. Moreover, most therapeutic having been the proof of their effectiveness in this pathology is either heavy of consequences in particular infectious concerns or no available in our areas.

We proposed to illustrate the diagnostic and therapeutic problems of ASD in our context through the study of 3 observations put together in an internal medicine department of Dakar.

\section{Observations}

\subsection{Observation 1}

A 56 years old woman, schoolteacher, was addressed to our internal medicine department for febrile cervical adenopathies and arthralgias. As antecedents, she had asymmetrical peripheral inflammatory arthralgias of the large articulations (elbow, shoulder and especially knee) for 10 years evolving by push. Also she had been followed for 6 months for a trailing angina resistant to antibiotics having led to a tonsillectomy.

She presented 1 month before her admission a flu syndrome with cephalgias, intense asthenia, fever with night prevalence with shivers and sweats. A quasi-generalized pruriginous skin eruption accompanied these feverish episodes. It also described a persistence of a pharyngal pain with painful saw allowing and recrudescence of the arthralgias. This symptomatology justified several consultations without improvement. At her admission, the temperature was up to $40.9^{\circ} \mathrm{C}$ and a heart rate of $118 \mathrm{pulse} / \mathrm{min}$. The physical examination found cervical inflammatory adenopathies, mobiles and not fistulized. She presented also erythematous and maculopapular skin lesions located on face, neck, back (Figure 1) and the inner face of the thighs. There were neither articular tumefactions nor deformations. 


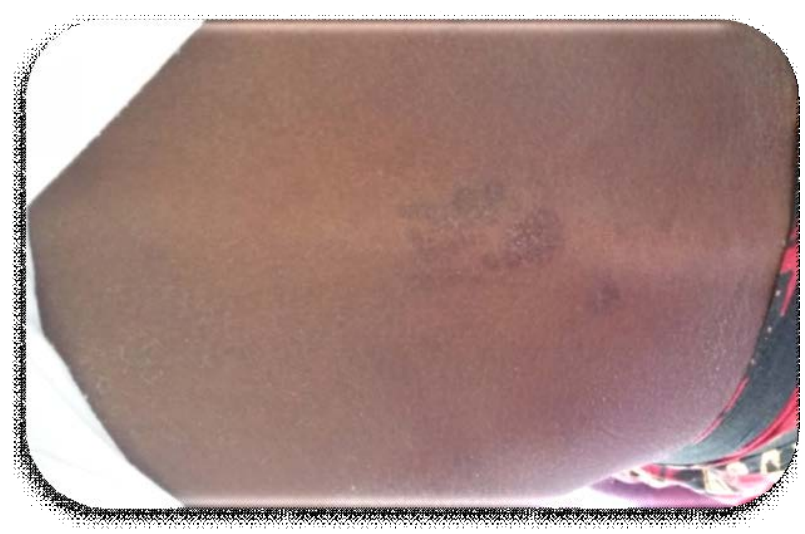

Figure 1. Erythematous and maculopapular skin lesions of the back.

The complementary examinations found hyperleucocytosis (total white cell count was $12.6 \times 10^{9} \mathrm{~g} / \mathrm{L}$ ) with $90 \%$ neutrophilia, anaemia (hemoglobin was 11.1 $\mathrm{g} / \mathrm{L}$ ) hypochromic mean corpuscular volume at $67 \mathrm{fl}$ (normal range $80-100 \mathrm{fl}$ ), a biological inflammatory syndrome, a moderate hepatic cytolysis and cholestasis. She presented a very high level of serum ferritin up to $20.948 \mathrm{mg} / \mathrm{L}$ (100 fold the normal range) with a low level of glycosylated fraction of ferritin at $16 \%$. All biological examination looking for infectious disease background remain negative: blood culture, cytobacteriologic examination of urines, test for malaria, search for mycobacterias on the phlegm, markers of viral hepatitis B and C, anti-VIH antibodies. Radiographies of the painful articulations were normal as well the thoraco-abdominal tomodensitometry. The research of rheumatoid factor, antinuclear and anti CCP antibodies was also negative. All in all, our patient presented six criteria out of eight for the Yamaguchi's criteria [4] as well for those of Fautrel [5], thus making us retain the diagnosis of ASD.

She has been treated with $0.8 \mathrm{mg} / \mathrm{kg} /$ day of prednisone. This treatment had allowed the fast disappearance of cutaneous lesions, arthralgias and progressive normalization of the biological anomalies. However, it was noted the appearance of muscular weakness of the 4 members quickly progressive having required a degression of the corticosteroid therapy. The patient complained a reappearance of the arthralgias then. In front of this situation we introduced methotrexate with the weekly dose of $20 \mathrm{mg}$ associated with folic acid and continuation of the progressive decrease of corticoids up to the daily dose of $2.5 \mathrm{mg}$ of prednisone. This biotherapy allowed the stable and complete disappearance of the whole of symptomatology and the normalization of the serum level of ferritin. All the treatment has been completely stopped 9 months later without any relapse so far.

\subsection{Observation 2}

A 30 years old woman without particular pathological antecedents was referred to our department for exploration of an unexplained prolonged fever. She had been hospitalized for 6 weeks in a hospital for a symptomatology which had evolved 
for 2 months. It was made of a permanent fever without shiver or sweat; but associated with a hepatomegaly and arthritis of the knee, ankle and of the wrist. This evolved in a context of deterioration of the general state. Several examinations with infectious aiming (thick drop, haemocultures, cytobacteriologic examination of urines) were carried out without any positive result. She had received before treatment against malaria and several protocols of antibiotic therapy without improvement of the symptomatology.

At admission in our department, the examination found febrile jaundice with fever at $39.5^{\circ} \mathrm{C}$ associated with sensitive regular hepatomegaly. There was a diffuse cutaneous xerosis, an acquired ichthyosis on the lower extremities (Figure 2) and pruritic cupboard on the back.

The joints of the wrist and the knees were tumefied, inflammatory with presence of an articular outpouring whose puncture brought back a viscous yellow liquid rich with no altered neutrophile polynuclear. The peripheral ganglionic areas were free and spleen was not palpable.

The biological examinations revealed an aregenerative pancytopenia, an inflammatory syndrome with high level of CRP at $192 \mathrm{mg} / \mathrm{l}$ (reference $<6 \mathrm{mg} / \mathrm{l}$ ), a cytolysis and a hepatic cholestasis. The serum ferritin level was very high up with 80 -fold the normal range $(20.168 \mu \mathrm{g} / \mathrm{L})$. The search for autoantibodies (antinuclear, anti-CCP2) was negative. The rate of alpha fœto-protein was normal. At the same time, viral serologies for the HIV, B and C hepatitis and the search for tuberculosis by PCR remain negative. The examination after medular ponction revealed an inflammatory aspect of bone marrow. The tomodensitometry found a magma of compressive adenopathies on the level of the cœlio-mesenteric and latero-aortic chains with inflammatory shape. The diagnosis of ASD probably associated with a syndrome of lymphohistiocytic activation was then strongly suspected.

She was put under bolus of methylprednisolone during 3 days followed by a relay with prednisone at a rate of a $1 \mathrm{mg} / \mathrm{kg} / \mathrm{day}$. She had also received iterative transfusions of concentrated platelet and packed red blood cell besides the additive treatment of the corticosteroid therapy. The evolution was marked after one short period of apyrexia, by febrile peaks reaching $41^{\circ} \mathrm{C}$, associated with a cardiovascular

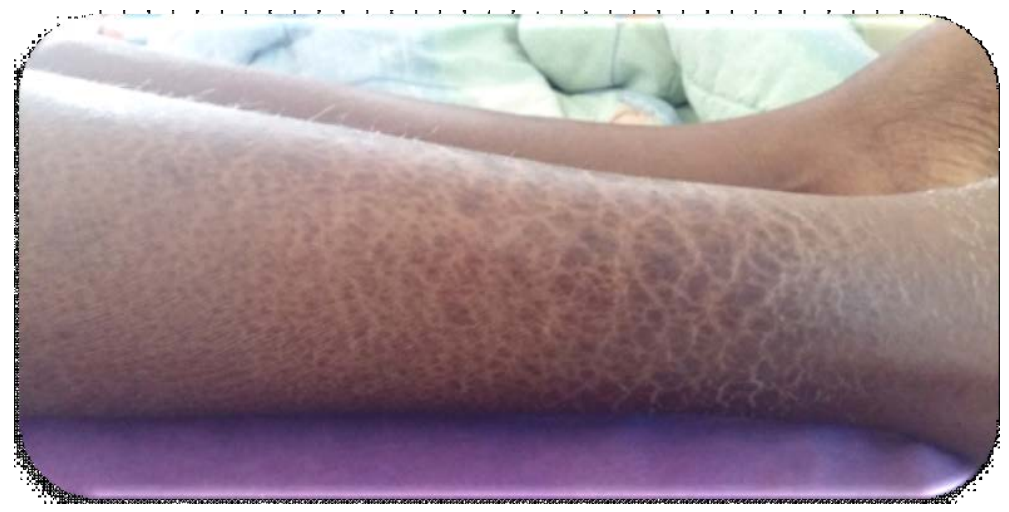

Figure 2. Diffuse cutaneous xerosis and acquired ichthyosis of the legs. 
collapse. Strong corticosteroid amounts were then maintained besides the symptomatic treatment. Methotrexate or immunosuppressant agents could not have been used because of hematologic instability and lack of result of different test performed to check for infectious disease. Moreover, hemocultures carried out during the second week of hospitalization had isolated Providencia stuartii in nosocomial context. An antibiotherapy adapted to the antibiogramme was then quickly performed besides the corticosteroid therapy. In spite of this treatment, the patient presented one week after a persistence of the hectic fever (Figure 3), generalized tonic-clonic convulsions, a right pleurisy (Figure 4) rich with non alterered neutrophiles polynuclear.

No other germ was identified in hemoculture, neither in urines nor in pleural and lumbar liquids. She died 6 days later in a context of multi visceral failure. The results of the dosage of glycosylated fraction of ferritin came back to us later showing low level at $18 \%$. It thus validated the ASD's criteria of Yamaguchi and Fautrel [4] [5] with a score of 5/8 for each classification.

\subsection{Observation 3}

This observation is about a young Guinean student, 22 years old. He had presented for 3 months an inflammatory bilateral localised to the shoulder, wrist, ankle and hip. Those symptoms evolved in a context of intermittent fever with shivers. This fever persisted in spite of a prior presumptive antimalarial treatment, antibiotics and paracetamol prescribed in his country before reaching our department in Dakar. It was associated with a progressive deterioration of his general state.

At the admission, he presented fever at $39^{\circ} \mathrm{C}$ and tachycardia at $108 \mathrm{pulse} / \mathrm{min}$. The physical examination was normal apart from an exacerbate pain while mobilizing the painful articulations. The complementary exploration highlighted an acceleration of VS at $58 \mathrm{~mm}$, a rate of CRP $15.5 \mathrm{mg} / \mathrm{L}(\mathrm{N}<6)$, and also discreet thrombocytosis and lymphopenia. The rate of white globules was normal. The serum protide electrophoresis showed a hypo albuminemia at $26.3 \mathrm{~g} / \mathrm{L}(\mathrm{N}=30$ - 40)

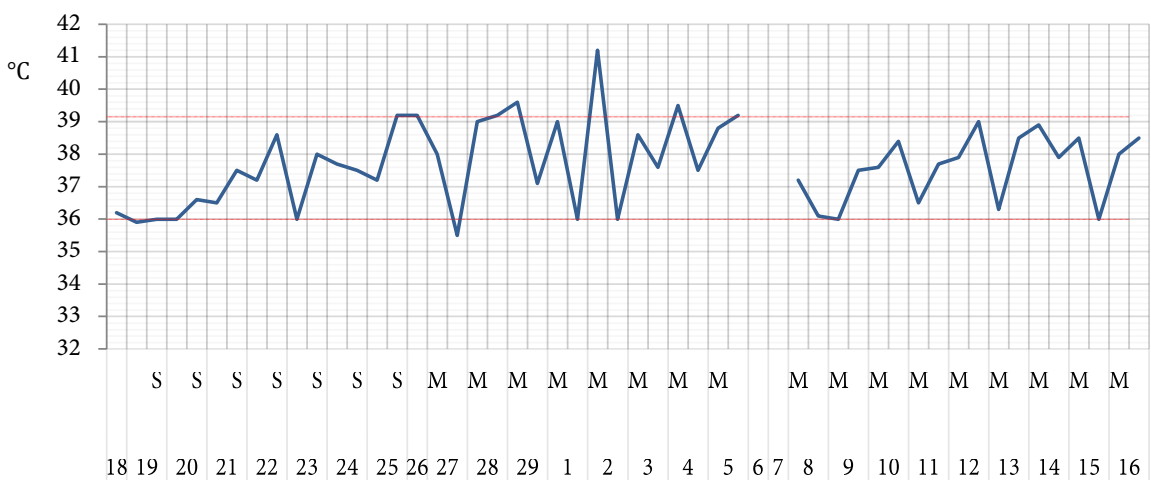

Février-Mars 2016

Figure 3. Hectic fever curve during hospitalization. 


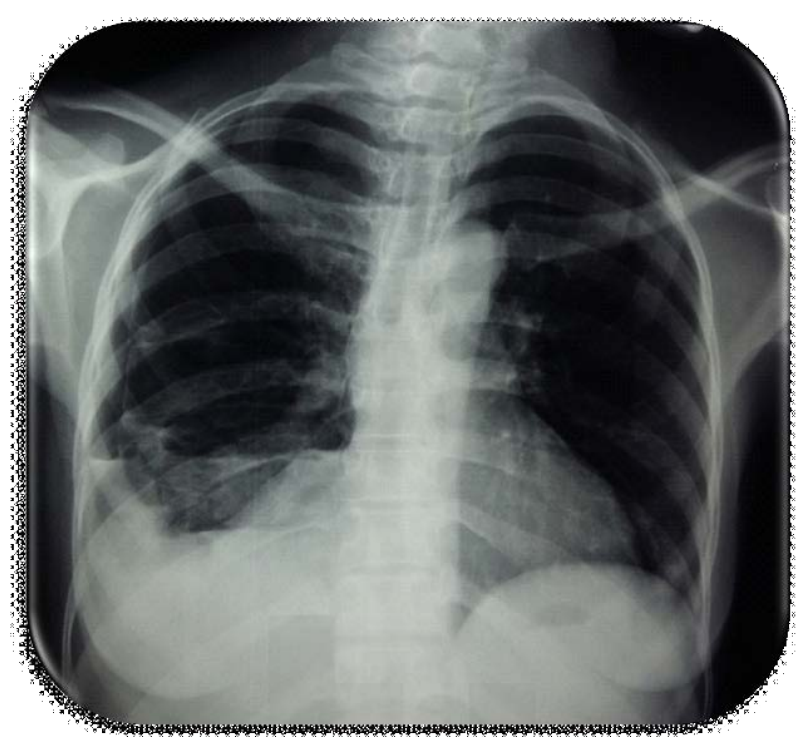

Figure 4. Right basal pleurisy.

and an increase diffuses $\gamma$-globulines. There was a hepatic cytolysis with 4 fold increase of ASAT/ASAT. We noticed a hyperferritinemia up to 4 fold $(1120 \mu \mathrm{g} / \mathrm{L})$ and the glycosylated fraction of ferritin was low at $42 \%$, but under corticosteroid therapy and delayed blood sampling because of lack of financial ressources. The repeated hemocultures were negative. Abdominal and cardiac ultrasound examinations were normal. The later research of the rheumatoid factor and common auto-antibodies was negative.

The diagnosis of ASD was retained early for this patient and $0.85 \mathrm{mg} / \mathrm{kg} / \mathrm{day}$ of prednisone was begun. It had allowed a rapid and stable apyrexia, a progressive improvement of arthralgias and general state as well a normalization of the biological disturbances. The initial amount of prednisone was gradually decreased until an amount of maintenance of $5 \mathrm{mg} /$ day reached in the 4 th month of treatment. The evolution had not ceased improving and the general state had been restored. He stopped the treatment 8 months after the hospitalization without relapse so far.

\section{Discussion}

ASD is a polygenic systemic disease of unknown cause [6]. In sub-Saharan Africa, works on ASD are summarized with some reports of case [2] [3]. In the majority of these African observations, it is noted a clear prevalence of female as found in our study. However, in the literature the sex-ratio currently tends to balance and the female prevalence seem to interest the chronic articular forms [1].

Indeed, there exist two phenotypes distinct of ASD. A systemic form with noisy polymorphic symptomatology exposing to the severe complications in particular the lymphocytic activation, and a chronic articular form of indolent evolution associated with the presence of arthritis being able to be destroying [1]. Our study is representative of these two clinical forms. The second patient presented the systemic 
form while the two others had a chronic articular form.

The diagnosis of ASD is based on a cluster of arguments. On the clinical level, the articular attack primarily related to the knees, ankles and wrists was noted among all our patients. The cutaneous lesions found for 2 patients among 3 were variable. Generally, it is described in ASD a macular skin eruption evanescent localized on the trunk and the root of the members [1] [6].

Our $2^{\text {nd }}$ patient presented however atypical cutaneous manifestations in the form of acquired ichthyosis and pruritic cupboard. Pruritic papules and/or plaques are recently reported in cases of ASD [7]. Most patients in this study had persistent and severe ASD, with a considerable frequency of clinical complications and reactive hemophagocytic syndrome as found in our $2^{\text {nd }}$ observation [7].

In the same way, the neurological attack in the forms of generalized convulsive crises found at the 2nd observation is seldom described during ASD [8].

The most typical hematologic abnormality during ASD is hyperleucocytosis with polynuclear neutrophile [1]. At the opposite, leucopenia being integrated within the framework to a pancytopenia was found in the second case thus making us think of an association with a lymphocytic activation syndrome. Indeed, the presence of cytopenia or the absence of hyperleucocytosis is elements of orientation towards a lymphocytic activation syndrome during ASD [9] [10]. The medullar ponction did not allow to find hemophagocytosis, however in our case. In a series of 52 patients with ASD, 8 presented a reactive hemophagocytic syndrome [11].

Moreover, all our three patients presented a hyperferritinemia higher than $1000 \mu \mathrm{g} / \mathrm{L}$ with reduction of the glycosylated fraction of ferritin. The fall of the glycosylated fraction of ferritin below $20 \%$ is a major diagnostic marker of MSA with a specificity of $93 \%$ [12]. Glycosylated fraction of ferritin was available in our context but not affordable for our patients with a delay of 4 to 8 weeks before obtaining the results.

In addition, it was noted in all our observations a diagnostic wandering. Our patients attended several medical structures before the diagnosis. They also received various anti-infectious non-successful treatments. This situation is source of important diagnostic delay noticed in most cases reported in sub-Saharan Africa [2] [3] but also in the whole world [13]. This delay is explained partly by the difficulty of differential diagnosis in front of suspected ASD which remain a diagnosis of elimination. Specifically, infectious pathologies must initially be excluded especially in tropical zone making the diagnosis more difficult.

The therapeutic strategy in ASD is still empirical. The first line corticosteroid therapy was efficient for two patients. The acute cortisonic myopathy as side-effects of the corticosteroid therapy are frequently reported and the early addition of cortisonic savings treatment can prevent it [1]. In this case, the introduction of methotrexate allows cortisonic savings with control of articular symptomatology. This molecule is recognized as being the first relay with empirical corticosteroid treatment in this aim [14]. It should be noted that in this systemic form, the blocking of the way of the interleukine-1 by using anakinra is effective, in the case 
of cortico-dependence [15]. These therapies are not accessible in our context.

\section{Conclusion}

ASD is a rare pathology but whose prevalence is probably under estimated in sub-Saharan Africa. Its nonspecific clinical presentation, lack of pathognomonic diagnostic biomarker, and the obligatory exclusion of systemic infectious pathologies before retaining the diagnosis explain the diagnostic difficulties of this pathology in particular in our tropical context. The treatment is not therefore easy with the side-effects of the corticosteroid therapy, optionally associated with methotrexate, but also the inaccessibility of the biotherapies in our areas.

\section{References}

[1] Gerfaud-Valentine, M., Sap, P., Hot, A., Broussolle, C. and Jamilloux, Y. (2015) Data Brought up to Date on Physiopathology, Phenotypes and Treatments of the Disease of Still of the Adult. Revue de Médecine Interne, 36, 319-327.

[2] Diallo, S., Pouye, A., Ndongo, S., Fall, S. and Diop, T. (2008) Disease of Still of the Adult: Study of 10 Senegalese Observations. Revue Du Rhumatisme, 75, 1105.

[3] Akintayo, R. and Adelowo, O. (2015) Adult-Onset Still's Disease in a Nigerian Woman. BMJ Case Reports. https://doi.org/10.1136/bcr-2015-210789

[4] Yamaguchi, M., Ohta, A., Tsunematsu, T., et al. (1992) Preliminary Criteria for Classification of Adult Still's Disease. The Journal of Rheumatology, 19, 424-430.

[5] Fautrel, B., Zing, E., Golmard, J., et al. (2002) Proposal for a New Set of Classification Criteria for Adult-Onset Still Disease. Medicine (Baltimore), 81, 194-200. https://doi.org/10.1097/00005792-200205000-00003

[6] Efthimiou, P., Paik, P.K. and Bielory, L. (2006) Diagnosis and Management of Adult Onset Still's Disease. Annals of the Rheumatic Diseases, 65, 564-572. https://doi.org/10.1136/ard.2005.042143

[7] Narvaez Garcia, F.J., Pascual, M., Lopez de Recalde, M., et al. (2017) Adult Onset Still's Disease with Atypical Cutaneous Manifestations. Medecine (Baltimore), 96, e6318. https://doi.org/10.1097/MD.0000000000006318

[8] Gallou, T., Gendrot, A., Clement, C., et al. (2009) Disease of Still of the Adult and Neurological Attack. Revue de Médecine Interne, 30, S465.

[9] Michota, J.M., Tamper, M., Galicier, L., et al. (2013) The Syndrome of Lymphohistiocytic Activation of the Adult. Revue de Médecine Interne, 34, 85-93.

[10] Rist, S., Manceron, V., Bartolucci, P., et al. (2005) Disease of Still and Leucopenia: In Connection with Two Observations. Revue de Médecine Interne, 26, 670-672. https://doi.org/10.1016/j.revmed.2005.04.031

[11] Hot, A., Toh, M.L., Coppéré, B., et al. (2010) Reactive Hemophagocytic Syndrome in Adult-Onset Still Disease: Clinical Features and Long-Term Outcome: A Case-Control Study of 8 Patients. Medicine (Baltimore), 89, 37-46. https://doi.org/10.1097/MD.0b013e3181caf100

[12] Fautrel, B., Moël, G., Saint-Marcoux, B., et al. (2001) Diagnosis Been Worth Of ferritin and Glycosylated Ferritin in Adult Onset Still's Disease. The Journal of Rheumatology, 28, 322-329.

[13] Park, S. and Pham, C. (2017) Delay in the Diagnosis of Adult-Onset Still's Disease. Cureus, 9, 1321. 
[14] Fautrel, B., Borget, C., Rozenberg, S., et al. (1999) Corticosteroid Sparing Effect of Low Amount Methotrexate Treatment in Adult Still's Disease. The Journal of Rheumatology, 26, 373-378.

[15] Moulis, G., Sailler, L., Astudillo, L., Pugnet, G. and Arlet, P. (2010) May Anakinra Be Used Earlier in Adult Onset Still Disease? Clinical Rheumatology, 29, 1199-1200. https://doi.org/10.1007/s10067-010-1459-6 